\title{
Recent Developments in the Rapid Diagnosis of MDR-TB
}

\section{Suhail Ahmad}

Department of Microbiology, Faculty of Medicine, Health Sciences Center, Kuwait University, Kuwait

*Corresponding author: Suhail Ahmad, Department of Microbiology, Faculty of Medicine, Health Sciences Center, Kuwait University, Kuwait, Tel: 00965-24636503; Email: suhail_ah@hsc.edu.kw

Received date: June 02, 2018; Accepted date: June 04, 2018; Published date: June 08, 2018

Copyright: @2018 Ahmad S. This is an open-access article distributed under the terms of the Creative Commons Attribution License, which permits unrestricted use, distribution, and reproduction in any medium, provided the original author and source are credited.

\section{Editorial}

Tuberculosis (TB) is a major infectious disease causing considerable morbidity and mortality among the most vulnerable individuals around the globe. Most active TB disease cases in humans are caused by Mycobacterium tuberculosis [1]. Active TB disease typically results from recent infection/re-infection in low-income, high TB burden countries while in high income, low TB incidence countries, it is usually caused by reactivation of a previously acquired infection (reactivation of latent infection) [1,2]. Despite declining trends in TB disease incidence and deaths in the past few years, the disease burden is still enormous. According to the recent WHO estimates, there were 10.4 million active TB disease cases (including 1 million patients coinfected with HIV) in 2016 which killed 1.7 million people worldwide [3]. The largest number (45\%) of disease cases occurred in South-East Asia region with five countries accounting for $\sim 56 \%$ of all TB cases [3]. The incidence varies considerably around the globe and is usually low $(<10$ cases per 100000 people ) in most high-income/low TB incidence countries but is very high (150-300 cases per 100000 people) in TB endemic countries [3].

The growing incidence of drug-resistant (DR)-TB, particularly, multidrug-resistant (MDR)-TB (M. tuberculosis resistant at least to rifampicin and isoniazid, the two most effective first-line drugs) and extensively drug-resistant (XDR)-TB (MDR-TB strains additionally resistant to a fluoroquinolone plus injectable agent, kanamycin, amikacin or capreomycin) is the main hindrance in controlling the global TB epidemic [3]. An estimated 600000 and 490000 new TB cases had rifampicin-resistant (RR)-TB and MDR-TB, respectively, causing nearly 240000 deaths in 2016 [3]. Nearly $91 \%$ of all RR/MDRTB cases occurred in the 40 high RR/MDR-TB burden countries in 2016 [3]. According to WHO, RR-TB cases also require the same treatment approaches as MDR-TB. It is estimated that nearly $4 \%$ of all new TB cases and nearly $19 \%$ of previously treated cases had MDR-TB with three countries accounting for 47\% of MDR-TB cases in 2016 [3]. According to recent estimates, about $10 \%$ of all MDR-TB cases now have XDR-TB [3].

Compared to the disease with drug-susceptible tubercle bacilli, treatment of patients with RR-TB and MDR/XDR-TB is difficult due to long (12-24 months) duration of treatment with costly and toxic drugs and the patients more frequently experience clinical failure or the disease relapses $[4,5]$. The treatment success rates of $83 \%, 54 \%$, and $30 \%$ have been reported for drug-susceptible TB, MDR-TB and XDR$\mathrm{TB}$, respectively [3]. The main reasons for poor treatment outcome for MDR/XDR-TB are the long duration of treatment and the nonavailability of new anti-TB drugs. To overcome these limitations, the WHO has recently introduced shorter (9-12 months) multidrug regimens for the treatment of RR-TB and MDR-TB in many poor, developing countries of Asia and Africa. The short regimens have achieved a treatment success rate of nearly $90 \%$ [3]. Two new anti-TB drugs; bedaquiline and delamanid have also been included in drug regimens in many countries for improved treatment outcome for MDR/XDR-TB [3]. Accurate and reliable drug susceptibility testing (DST) of $M$. tuberculosis to first-line (rifampicin, isoniazid, ethambutol and pyrazinamide) drugs is required for the diagnosis of DR-TB and MDR-TB and for effective management of TB patients $[4,6,7]$. Effective treatment will also limit further development of resistance to other anti-TB drugs leading to XDR-TB [4]. Recent modelling studies have suggested that improper management of patients with DR/MDR-TB may lead to replacement of drugsusceptible TB by MDR-TB as the dominant phenotype across the world [8]. Thus rapid diagnosis of MDR-TB is crucial for proper patient management.

Phenotypic DST was considered as the most reliable laboratory approach to determine susceptibility or resistance of $M$. tuberculosis due to good clinical correlation and quality control until recently. Phenotypic DST by solid (Lowenstein-Jensen or 7H10 agar) mediumbased critical proportion method is considered as the gold standard for first-line (except pyrazinamide) and important second-line (new generation fluoroquinoloes and injectable agents; kanamycin, amikacin or capreomycin) drugs. However, the method is very slow as it requires nearly six weeks to report results [6,7]. To overcome this limitation, commercial liquid culture systems and genotypic assays have been developed for more rapid detection of drug resistance in $M$. tuberculosis [4,7]. The liquid-broth-based fully automated Mycobacteria Growth Indicator Tube (MGIT) 960 system was developed as a rapid phenotypic DST method as it reported results within 10-14 days [6,7]. Although the performance of this liquidmedium-based method has been excellent for isoniazid, some studies have shown poor performance for M. tuberculosis isolates for the other three (rifampicin, pyrazinamide and ethambutol) first-line drugs for different reasons [9].

Resistance of $M$. tuberculosis to rifampicin in $\sim 97 \%$ isolates is due to mutations in hot-spot region of the $r p o B$ gene [4,9]. The solid medium-based method with shorter ( 4 weeks) turnaround time and the liquid broth-based MGIT 960 system frequently fail to detect rifampicin resistance in $M$. tuberculosis strains exhibiting low-level (minimum inhibitory concentration, MIC of $0.5 \mu \mathrm{g} / \mathrm{ml}-2.0 \mu \mathrm{g} / \mathrm{ml}$ ) resistance [10-12]. These isolates contain specific $r p o B$ mutations (disputed $r p o B$ mutations) which may account for up to $10 \%$ of all such mutations in $M$. tuberculosis isolates from some geographical locations and the patients infected with these strains often fail treatment or the disease relapses $[9,10,13]$. Pyrazinamide is another key drug for the treatment of MDR-TB but resistance to pyrazinamide is also found frequently among MDR-TB strains $[3,6,9]$. Phenotypic testing often yields unreliable DST results as it requires precise acidic conditions $(\mathrm{pH} 5.6)$ which prevent the growth of nearly $20 \%$ of the isolates while larger inoculum, if added, during DST leads to higher $\mathrm{pH}$ of the medium causing false pyrazinamide resistance $[6,9]$. 
Ethambutol is a bacteriostatic anti-TB drug and problems with accurate phenotypic DST for ethambutol are well-known, particularly with rapid liquid culture-based methods $[6,14]$. The main targets of ethambutol are three arabinosyltransferase genes encoded by embCAB operon. Mutations in embCAB operon occur first, are clinically significant and mostly involve embB, particularly at codons 306, 406 and 497. However, these mutations increase the MIC by only 3-8 folds (close to the critical concentration of the drug) and so are often missed by rapid liquid culture systems $[4,6,14]$. It is pertinent to mention here that false susceptibility to ethambutol is important only for the treatment of RR-TB and MDR/XDR-TB $[6,15]$. Phenotypic methods yield accurate results for streptomycin and second-line drugs; fluoroquinolones and injectable drugs (kanamycin, amikacin and capreomycin) $[6,7,15]$. The phenotypic DST methods for less active second-line and other drugs are usually inadequate mainly because the critical concentrations for these agents are not well-defined, the methods have not been standardized internationally and so are not completely reliable $[6,7,9]$.

The problems associated with slow and/or inaccurate phenotypic DST of $M$. tuberculosis can be circumvented by using molecular methods. These methods typically detect genetic mutations associated with drug resistance rapidly (1-2 days) and so shorten the time between MDR/XDR-TB diagnosis and institution of appropriate treatment regimens [7,16,17]. Furthermore, different mutations in target genes confer different levels of phenotypic resistance and some mutations increase the odds of patient mortality [9]. Molecular methods mainly include hybridization/reverse hybridization-based assays, PCR amplification followed by direct sequencing of PCR products for select panel of target genes and whole-genome sequencing (WGS) for TB bacilli in clinical specimens and culture isolates [9].

Hybridization-based assays include GeneXpert MTB/RIF assay (GeneXpert), line probe assays and various formats of DNA microarrays for the detection of resistance to different first-line and/or second-line drugs. GeneXpert is a fully automated, cartridge-based point-of-care real-time PCR assay that detects presence of $M$. tuberculosis in clinical specimens and its resistance to rifampicin [18]. The method also detects $\sim 85 \%$ of MDR-TB cases $[4,18]$. Another cartridge-based point-of-care test has recently been developed for simultaneous detection of resistance to isoniazid, fluoroquinolones and second-line injectable agents [19]. Both these rapid tests help in the diagnosis of XDR-TB. One disadvantage of the GeneXpert (and other hybridization-based assays) is the recent detection of silent mutations in the $r p o \mathrm{~B}$ gene which lead to false rifampicin resistance detection by this test [12].

Line probe assays mainly include GenoType MTBDRplus assay that detects resistance of $M$. tuberculosis to rifampicin and isoniazid (for the detection of MDR-TB) and GenoType MTBDRsl assay that detects resistance to fluoroquinolones (ofloxacin, levofloxacin etc.) and injectable agents (kanamycin, amikacin and capreomycin) in MDR-TB strains for the diagnosis of XDR-TB [4,9]. Similar to GeneXpert assay, line probe assays are also prone to report false drug resistance detection due to presence of synonymous point mutations in target region [12]. DNA microarrays have also been developed for the detection of resistance to different anti-TB drugs. A commercial DNA microarray (GeneChip) and a simplified microarray test identify mutations in $r p o B, k a t G+i n h A, e m b B$, and $r p s L$ for the detection of drug resistance to anti-TB drugs including the diagnosis of MDR-TB [9]. An integrated microfluidic card with TaqMan probes and highresolution melt analysis has been developed recently that detects resistance of $M$. tuberculosis to rifampicin, isoniazid, pyrazinamide, ethambutol, fluoroquinolones and injectable drugs [9].

PCR amplification of target region followed by direct DNA sequencing of PCR amplicons detects resistance to different anti-TB drugs by interrogating appropriate number and regions of loci conferring resistance to these agents $[16,17]$. However, the sensitivity of this approach varies considerably since multiple loci are involved in conferring resistance to some anti-TB drugs and thus depends on the number of loci included for testing. The sensitivity is also affected by the varying frequency of different mutations in these loci at different geographical locations or among M. tuberculosis strains isolated from patients of different ethnic groups [4,20]. This approach is, however, tedious and time consuming as it requires screening of multiple gene loci for many anti-TB drugs and so is being replaced by WGS [21].

The WGS characterizes both frequently encountered and rarely detected mutations predicting drug resistance for all anti-TB drugs (including new drugs, bedaquilline and delamanid). The coverage of the entire genome also makes WGS a rapidly scalable method for determination of drug resistance caused by any chromosomal mutation to first-line and second-line drugs as well as newer anti-TB agents to inform treatment [21]. Recent studies have also shown the use and application of WGS for tracking transmission and outbreaks of DR-TB and MDR-TB and for identifying novel mechanisms of drug resistance [22]. The WGS of $M$. tuberculosis can be performed directly on clinical samples from TB patients to rapidly generate antibiotic susceptibility profiles for same day diagnosis [23].

Two European countries (England and Italy) are now using WGS on a national scale to realize its full potential for rapid diagnosis of TB, detection of resistance to anti-TB drugs, and fingerprinting of $M$. tuberculosis for contact investigation and continuous surveillance $[24,25]$. However, the method is costly and also requires technical expertise and bioinformatic support. Due to these reasons, WGS is still difficult to implement in resource-poor TB endemic countries. Costeffective and simplified ways of WGS data acquisition and analysis are urgently needed for poor/developing TB endemic countries to achieve WHO's target of 'End TB by 2035 .

\section{Acknowledgements}

Research support provided by Kuwait University Research Sector through grant MI03/16 is gratefully acknowledged.

\section{References}

1. Ahmad S (2010) New approaches in the diagnosis and treatment of latent tuberculosis infection. Resp Res 11: 169.

2. Mokaddas E, Ahmad S, Samir I (2008) Secular trends in susceptibility patterns of Mycobacterium tuberculosis isolates in Kuwait, 1996-2005. Int J Tuberc Lung Dis 12: 319-325.

3. WHO (2017) Global tuberculosis report 2017. Geneva, Switzerland.

4. Ahmad S, Mokaddas E (2014) Current status and future trends in the diagnosis and treatment of drug-susceptible and multidrug-resistant tuberculosis. J Infect Public Health 7: 75-91.

5. Dheda K, Gumbo T, Maartens G, Dooley KE, McNerney R, et al. (2017) The epidemiology, pathogenesis, transmission, diagnosis, and management of multidrug-resistant, extensively drug-resistant, and incurable tuberculosis. Lancet Respir Med 5: 291-360.

6. Horne DJ, Pinto LM, Arentz M, Lin SY, Desmond E, et al. (2013) Diagnostic accuracy and reproducibility of WHO-endorsed phenotypic drug susceptibility testing methods for first-line and second-line antituberculosis drugs. J Clin Microbiol 51: 393-401. 
7. Schön T, Miotto P, Köser CU, Viveiros M, Böttger E, et al. (2017) Mycobacterium tuberculosis drug-resistance testing: challenges, recent developments and perspectives. Clin Microbiol Infect 23: 154-160.

8. McBryde ES, Meehan MT, Doan TN, Ragonnet R, Marais BJ, et al. (2017) The risk of global epidemic replacement with drug-resistant Mycobacterium tuberculosis strains. Int J Infect Dis 56: 14-20.

9. Ahmad S, Mokaddas E (2018) Recent advances in proper management of multidrug-resistant tuberculosis. Kuw Med J 50: 146-160.

10. Van Deun A, Aung KJ, Bola V, Lebeke R, Hossain MA, et al. (2013) Rifampin drug resistance tests for tuberculosis: challenging the gold standard. J Clin Microbiol 51: 2633-2640.

11. Ahmad S, Mokaddas E, Al-Mutairi N, Eldeen HS, Mohammadi S (2016) Discordance across phenotypic and molecular methods for drug susceptibility testing of drug-resistant Mycobacterium tuberculosis isolates in a low TB incidence country. PLoS One 11: e0153563.

12. Mokaddas E, Ahmad S, Eldeen HS, Al-Mutairi N (2015) Discordance between Xpert MTB/RIF assay and Bactec MGIT 960 culture system for detection of rifampin-resistant Mycobacterium tuberculosis isolates in a country with a low tuberculosis (TB) incidence. J Clin Microbiol 53: 1351-1354.

13. Van Deun A, Aung KJ, Hossain A, de Rijk P, Gumusboga M, et al. (2015) Disputed rpoB mutations can frequently cause important rifampicin resistance among new tuberculosis patients. Int J Tuberc Lung Dis 19: 185-190.

14. Ahmad S, Jaber AA, Mokaddas E (2007) Frequency of embB codon 306 mutations in ethambutol-susceptible and -resistant clinical Mycobacterium tuberculosis isolates in Kuwait. Tuberculosis 87: 123-129.

15. Al-Mutairi NM, Ahmad S, Mokaddas E (2018) Molecular screening versus phenotypic susceptibility testing of multidrug-resistant Mycobacterium tuberculosis isolates for streptomycin and ethambutol Microb Drug Resist.

16. Campbell PJ, Morlock GP, Sikes RD, Dalton TL, Metchock B, et al. (2011) Molecular detection of mutations associated with first- and second-line drug resistance compared with conventional drug susceptibility testing of
Mycobacterium tuberculosis. Antimicrob Agents Chemother 55: 2032-2041.

17. Al-Mutairi N, Ahmad S, Mokaddas E (2011) Performance comparison of four methods for rapid detection of multidrug-resistant Mycobacterium tuberculosis strains. Int J Tuberc Lung Dis 15: 110-115.

18. Boehme CC, Nabeta P, Hillemann D, Nicol MP, Shenai S, et al. (2010) Rapid molecular detection of tuberculosis and rifampin resistance. N Engl J Med 363: 1005-1015.

19. Xie YL, Chakravorty S, Armstrong DT, Hall SL, Via LE, et al. (2017) Evaluation of a rapid molecular drug-susceptibility test for tuberculosis. N Engl J Med 377: 1043-1054.

20. Ahmad S, Fares E, Araj GF, Chugh TD, Mustafa AS (2002) Prevalence of S315T mutation within the katG gene in isoniazid-resistant clinical Mycobacterium tuberculosis isolates from Dubai and Beirut. Int J Tuberc Lung Dis 6: 920-926.

21. Heyckendorf J, Andres S, Köser CU, Olaru ID, Schön T, et al. (2018) What is resistance? Impact of phenotypic versus molecular drug resistance testing on therapy for multi- and extensively drug-resistant tuberculosis. Antimicrob Agents Chemother 62: 15-17.

22. Arnold A, Witney AA, Vergnano S, Roche A, Cosgrove CA, et al. (2016) XDR-TB transmission in London: case management and contact tracing investigation assisted by early whole genome sequencing. J Infect 73: 210-218.

23. Votintseva AA, Bradley P, Pankhurst L, Del Ojo Elias C, Loose M, et al. (2017) Same-day diagnostic and surveillance data for tuberculosis via whole-genome sequencing of direct respiratory samples. J Clin Microbiol 55: 1285-1298.

24. Satta G, Lipman M, Smith GP, Arnold C, Kon OM, et al. (2018) Mycobacterium tuberculosis and whole-genome sequencing: how close are we to unleashing its full potential? Clin Microbiol Infect 24: 604-609.

25. Cabibbe AM, Trovato A, De Filippo MR, Ghodousi A, Rindi L, et al. (2018) Countrywide implementation of whole genome sequencing: an opportunity to improve tuberculosis management, surveillance and contact tracing in low incidence countries. Eur Respir J. 